\title{
MACHINE LEARNING BASED DATA RETRIEVAL FOR INVERSE SCATTERING PROBLEMS WITH INCOMPLETE DATA
}

\author{
YU GAO AND KAI ZHANG*
}

\begin{abstract}
We are concerned with the inverse scattering problems associated with incomplete measurement data. It is a challenging topic of increasing importance that arise in many practical applications. Based on a prototypical working model, we propose a machine learning based inverse scattering scheme, which integrates a CNN (convolution neural network) for the data retrieval. The proposed method can effectively cope with the reconstruction under limited-aperture and/or phaseless far-field data. Numerical experiments verify the promising features of our new scheme.
\end{abstract}

Keywords: Inverse scattering, limited aperture, phaseless, data retrieval, machine learning, convolution neural network

2010 Mathematics Subject Classification: 35Q60, 35J05, 31B10, 35R30, 78A40

\section{INTRODUCTION}

1.1. Background and motivation. Inverse scattering problems are concerned with the recovery of unknown/inaccessible objects from the corresponding wave probing data. They lie at the heart of many scientific and industrial developments including radar and sonar, medical imaging, geophysical exploration and non-destructive testing [3,10,11,13-15].

One class of inverse scattering problems that has received significant attentions recently in the literature is the inverse problems with incomplete data. Those problems could arise in various applications of great importance. The incomplete measurement data may be of limited aperture or/and without phase information. Various mathematical strategies have been proposed for retrieving the full wave data from the measured partial/incomplete data, and then they are integrated into the reconstruction process $[2,10,18,30,34,40,43,57,59,60]$. Generally speaking, if sufficient a-priori information is available on the underlying target scatterer, one may still be possible to achieve the recovery to a certain content level. Otherwise, the reconstruction shall suffer from great deterioration due to the intrinsic lack of information. However, some newly emerging applications may have more restrictive and higher quality requirements on the data retrieval and ultimately the recovery effects. In fact, it is noted that there is an increasing trend on designing portable and handheld medical devices that are fit for family use, say e.g. ultrasound or MRI scanners that can produce medical imaging on one's smart phone. Clearly, in addition to the hardware requirements, the core to the success of such conceptual applications would be the development of novel inverse scattering schemes that can work effectively and efficiently with highly incomplete measurement data. This motivates the current study by integrating the machine learning techniques into the data retrieval.

Machine learning methods, in particular the convolutional neural networks (CNNs), have been regarded as a revolutionary idea for many applied sciences. In fact, they have been successfully developed for dealing with various inverse problems with an increasing amount of literature. In this article, we propose a CNN-based data retrieval approach, which in 
combination with a sampling-type imaging scheme can produce stunning reconstructions for inverse scattering problems with limited-aperture or/and phaseless measurement data. Since it is rather impractical to consider too many different inverse scattering problems, we develop our method based on a prototypical model, the so-called inverse acoustic obstacle problem. The rest of this section is devoted to the introduction of this model inverse scattering problem as well as some relevant discussions on the reconstruction methods.

1.2. Mathematical setup and relevant discussions. Let $k=\omega / c \in \mathbb{R}_{+}$be the wave number of a time harmonic acoustic wave with $\omega \in \mathbb{R}_{+}$and $c \in \mathbb{R}_{+}$denoting, respectively, the angular frequency and the wave speed in a homogeneous background space. Let $\Omega \subset$ $\mathbb{R}^{n}(n=2,3)$ be a bounded domain with a Lipschitz-boundary $\partial \Omega$ such that its complement $\mathbb{R}^{n} \backslash \bar{\Omega}$ is connected. Let the incident field $u^{i}$ be a plane wave of the form

$$
u^{i}:=u^{i}(x, \hat{\theta}, k)=e^{i k x \cdot \hat{\theta}}, \quad x \in \mathbb{R}^{n},
$$

where $\hat{\theta} \in \mathbb{S}^{n-1}$ denotes the impinging direction of the incident wave and $\mathbb{S}^{n-1}:=\left\{x \in \mathbb{R}^{n}\right.$ : $|x|=1\}$ is the unit sphere in $\mathbb{R}^{n}$. In acoustic probing, one sends an incident wave $u^{i}$ to detect the unknown/inaccessible scatterer $\Omega$. The scatterer interrupts the propagation of the plane wave and generates the so-called scattered wave $u^{s}$. Set $u=u^{i}+u^{s}$ to denote the total wave field. The acoustic obstacle scattering is described by the following boundary value problem,

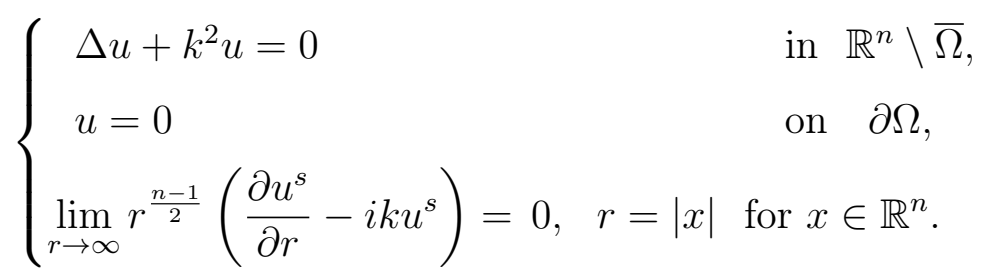

In (1.1), the homogeneous Dirichlet boundary condition on $\partial D$ signifies that $D$ is a soundsoft obstacle. The third equation is known as the Sommerfeld radiation condition, which characterizes the outgoing nature of the scattered wave field. The forward scattering problem is to compute $u^{s}$ (or $u$ ) for a given incident field $u^{i}$ and domain $D$. We refer to [14,52] for the well-posedness of the forward scattering problem (1.1) in $H_{\text {loc }}^{1}\left(\mathbb{R}^{n} \backslash \bar{D}\right)$.

In particular, one has the following asymptotic expansion of the scattered field,

$$
u^{s}(\Omega ; x, \theta)=\frac{e^{i \frac{\pi}{4}}}{\sqrt{8 k \pi}}\left(e^{-i \frac{\pi}{4}} \sqrt{\frac{k}{2 \pi}}\right)^{n-2} \frac{e^{i k r}}{r^{\frac{n-1}{2}}}\left\{u^{\infty}(\Omega ; \hat{x}, \hat{\theta})+\mathcal{O}\left(\frac{1}{r}\right)\right\} \quad \text { as } r \rightarrow \infty,
$$

which holds uniformly with respect to all observation directions $\hat{x}:=x /|x| \in \mathbb{S}^{n-1}$. The complex-valued function $u^{\infty}$ in (1.2) defined on the unit sphere $\mathbb{S}^{n-1}$ is known as the scattering amplitude or far-field pattern. The inverse scattering problem we are concerned with is to recover $\Omega$ by the knowledge of the far-field pattern

$$
u^{\infty}(\Omega ; \hat{x}, \hat{\theta}):(\hat{x}, \hat{\theta}) \in \Gamma \times \Sigma,
$$

where $\Gamma$ and $\Sigma$ are open subsets of $\mathbb{S}^{n-1}$ and are referred to as the observation aperture and incident aperture, respectively. If $\Gamma=\mathbb{S}^{n-1}$ and $\Sigma=\mathbb{S}^{n-1}$, then the corresponding inverse scattering problem is said to have full-aperture measurement data. Otherwise, it is said to have limited-aperture measurement data. By introducing an abstract operator $\mathcal{F}$ which sends the underlying obstacle to its corresponding far-field pattern and is defined by the Helmholtz 
system (1.1), the inverse problem can be formulated as the following operator equation,

$$
\mathcal{F}(\Omega)=u^{\infty}(\hat{x}, \hat{\theta}), \quad(\hat{x}, \hat{\theta}) \in \Gamma \times \Sigma .
$$

It can be verified that the inverse problem (1.3) is nonlinear. Moreover, it is severely illconditioned in the sense of Hadamard; that is, a small perturbation in the far-field data of (1.3) may cause a significant change in reconstructing $\Omega$ (cf. [16,47,54]).

It is known that the full-aperture data uniquely determines the obstacle $D$ (cf. [14]). Since $u_{\infty}(\hat{x}, \hat{\theta})$ is a real-analytic function on $\mathbb{S}^{n-1} \times \mathbb{S}^{n-1}$ (cf. [14]), the far-field pattern on $\Gamma \times \Sigma$ can be extended to the full-aperture data by unique continuation. Hence the limitedaperture data also uniquely determines the obstacle. However, it is well known that the analytic continuation is a severely ill-conditioned process (cf. [6]). Thus the inverse scattering problem suffers from an increasing level of ill-posedness as the size of aperture decreases. Indeed, this phenomenon has been observed and investigated in the existing literature, see e.g. $[1,2,7,13,29,41,46,50,51,53,62]$. In what follows we shall also present several numerical examples to reemphasize this point.

There is another scenario of practical importance where the measurement data is given by

$$
\mathcal{F}(\Omega)=\left|u^{\infty}(\Omega ; \hat{x}, \hat{\theta})\right|, \quad(\hat{x}, \hat{\theta}) \in \Gamma \times \Sigma .
$$

For the phaseless inverse problem (1.4), there is a well-known obstruction that one cannot determine the location of the obstacle $\Omega$. That is, if one shifts the obstacle to another location, the modulus of the corresponding far-field pattern remains unchanged. However, it is still possible to determine only the shape of the obstacle by knowing its position a priori (cf. [40,43]). Many inverse scattering schemes developed for phased measurement data in principle do not work for the phaseless case. Hence, the phaseless inverse scattering problem constitutes another challenging topic in the literature [18,30,31,40,43, 59,60]. As indicated in $[59,60]$, in the phaseless setup, it is physically more relevant to consider the measurement of the modulus of the total field on a sufficient large surface enclosing the obstacle other than the modulus of the far-field pattern. We would like to emphasize that the data retrieval method developed in what follows can be easily extended to work for the case with the modulus of the total wave field. However, in order to have a uniform formulation as well as to ease our exposition, we shall confine our study in the phaseless case with the measurement data given by (1.4).

The rest of the paper is organized as follows. In Section 2, we consider a samplingtype method for the inverse obstacle problem and show by a few numerical examples the intrinsic ill-posedness due to the lack of information. In Section 3, we briefly go through the main ingredients on CNN for our subsequent use. Section 4 is devoted to the algorithmic developments and numerical experiments.

\section{A SAMPLING-TYPE RECONSTRUCTION SCHEME AND ITS LOCALISED FEATURES}

The mainstream numerical methods for solving the inverse scattering problems (1.3) or (1.4) can be classified as sampling-type methods and optimization based iterative methods. Most of the sampling methods, e.g. MUSIC-type methods [3-5], linear sampling method [12] and factorization method [33], are designed to work with the full-aperture data. Some of them can be modified to cope with limited-aperture data, but only under special circumstances and suffer from the intrinsic ill-posedness. The optimization methods can be applied for any 
limited-aperture data as long as the objective functional is properly defined. The limitedaperture case has been studied by researchers using other methods, such as a regularized homotopy continuation method [7], a variant of the enclosure method [29], a generalization of the orthogonal projection method [53], and the methods based on transformed field expansion [46]. The methods for reconstructing polygonal and polyhedral obstacles also work with limited-aperture or even phaseless data [40,43].

Another approach for solving inverse scattering problems with limited aperture data is to first retrieve the missing data and then apply the existing numerical methods for the corresponding full-aperture problems. In the literature, the missing information retrieval is mainly based on the use of the intrinsic and generic structures of the far-field data. The aforesaid structures include the real-analyticity and reciprocity relations that hold generically for any set of far-field data, see e.g. [49]. There are also some strategies developed based on introducing an artificial reference scatterer into the scattering system for retrieving the phase information of the measurement data in the phaseless case $[18,30,31,44,59,60]$.

In what follows, we present a sampling-type method for the inverse scattering problem (1.3), and use several examples to discuss the intrinsic ill-posedness of (1.3) in the case with missing information. The core of the aforesaid method is the following imaging functional

$$
I(z):=\left|\int_{\Sigma} e^{-i k \hat{\theta} \cdot z} \int_{\Gamma} u^{\infty}(\hat{x}, \hat{\theta}) e^{i k \hat{x} \cdot z} d s(\hat{x}) d s(\hat{\theta})\right|^{2}, \quad z \in \mathbb{R}^{n} .
$$

The characteristic behaviour of the imaging functional (2.1) has been studied in [24,42, 45, 48,49 ], mainly in the case with full measurement data. Next, we present some numerical examples of the obstacle reconstruction by using the imaging functional (2.1) in the two dimensional case. The boundaries of the obstacles used in our numerical experiments are parameterized as follows:

$$
\begin{array}{cl}
\text { kite: } & x(t)=(a, b)+(\cos t+0.65 \cos 2 t-0.65,1.5 \sin t), \quad 0 \leq t \leq 2 \pi, \\
\text { round square: } & x(t)=(a, b)+9 / 4\left(\cos ^{3} t+\cos t, \sin ^{3} t+\sin t\right), \quad 0 \leq t \leq 2 \pi,
\end{array}
$$

with $(a, b)$ signifying the center of the obstacle which may be different in different examples. The plot of the regions centered at $(0,0)$ is shown in Figure 1.
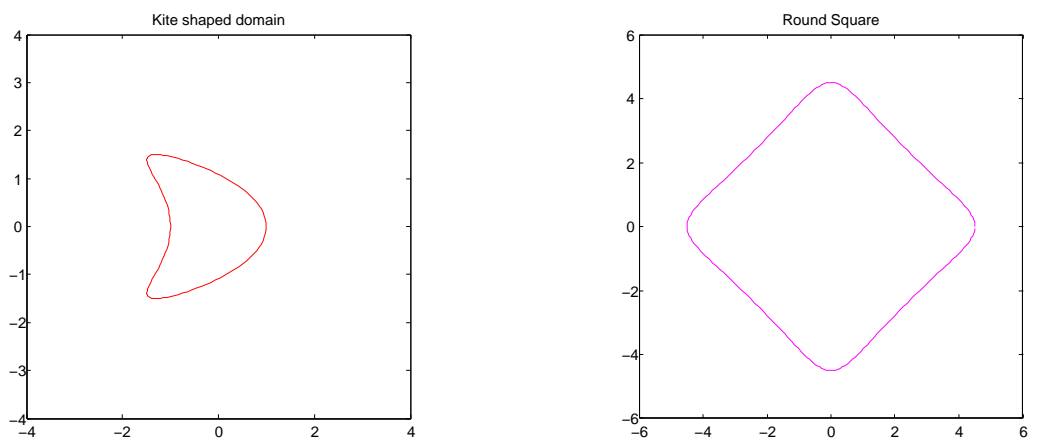

FigurE 1. True obstacles: kite shaped obstacle and round-square shaped obstacle.

Denote by $\hat{x}:=(\cos \phi, \sin \phi)$ the observation direction with angle $\phi \in(0,2 \pi)$. In the first example we consider the set $\left\{u^{\infty}(\hat{x}, \hat{\theta}): \hat{x} \in \Gamma, \hat{\theta} \in \mathbb{S}^{1}\right\}$ of far field patterns as data for the inverse problem, i.e., a limited observation aperture $\Gamma \subset \mathbb{S}^{1}$ and the full incident aperture 
$\Sigma=\mathbb{S}^{1}$. In Figures 2-3, we show the reconstructions of a kite shaped obstacle with different limited observation aperture $\Gamma$. As in many other numerical methods, see e.g. [41], a typical feature of the limited-aperture problems is that the "shadow region" is elongated in down range. However, Figures 2-3 show our results and confirm that, despite the use of the limited aperture data, the region $\partial D_{+}(\hat{x}):=\{y \in \partial D \mid \nu(y) \cdot \hat{x}>0\}$ with $\nu \in \mathbb{S}^{1}$ signifying the exterior unit normal vector to $\partial D$, which can be observed directly from the direction $\hat{x}$, is quite well captured. The imaging functional (2.1) produces a localized reconstruction of the obstacle. As seen in Figure 3, the quality of the reconstruction deteriorates as the aperture decreases. Physically, the information from the "shadow region" $\partial D_{-}(\hat{x}):=\{y \in \partial D \mid \nu(y) \cdot \hat{x}<0\}$ is very weak, especially for high frequency waves [50]. We also refer to Figure 4 for the reconstructions with the same observation aperture, but different wave numbers.

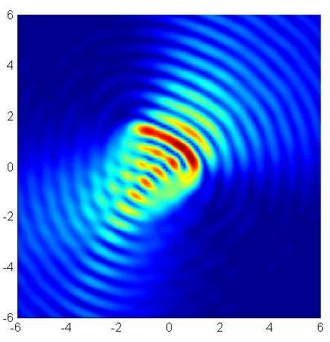

(a) $\phi \in(0, \pi / 2)$

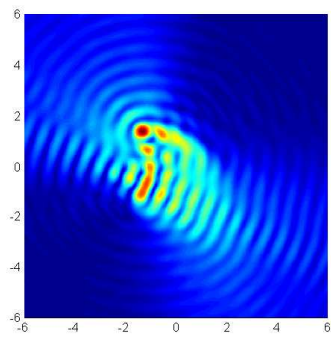

(b) $\phi \in(\pi / 2, \pi)$

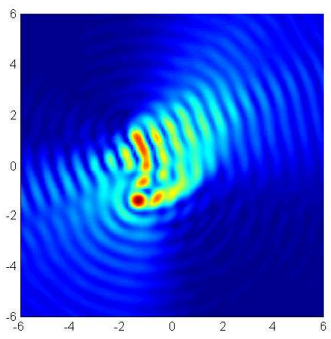

(c) $\phi \in(\pi, 3 \pi / 2)$

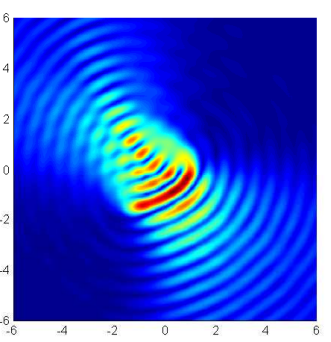

(d) $\phi \in(3 \pi / 2,2 \pi)$

FIGURE 2. Reconstructions of the kite shaped sound-soft obstacle with the wave number $k=5,10 \%$ noise and different observation apertures.

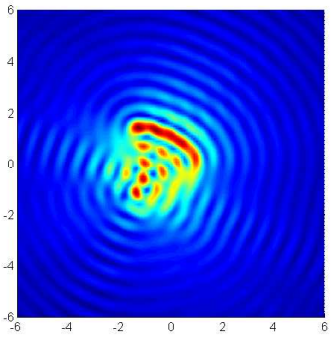

(a) $\phi \in(0, \pi)$

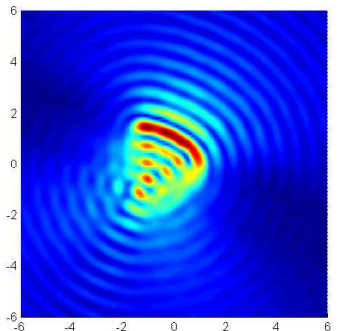

(b) $\phi \in(0,3 \pi / 4)$

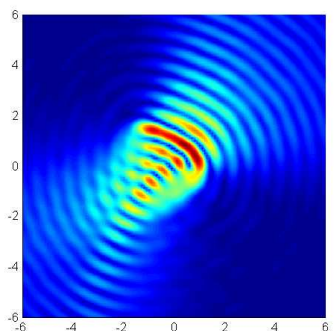

(c) $\phi \in(0, \pi / 2)$

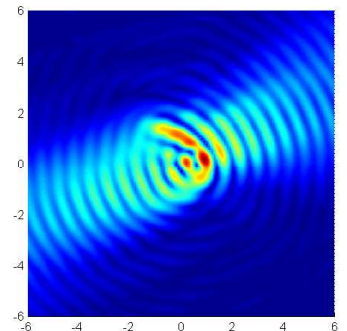

(d) $\phi \in(0, \pi / 4)$

FiguRE 3. Reconstructions of the kite shaped obstacle with the wave number $k=5,10 \%$ noise and different observation apertures.

In the following, we provide a partial theoretical explanation to the localized features of the reconstructions in the above numerical examples. For a sound-soft obstacle $D$, the far field pattern of the scattered field $u^{s}$ is given by

$$
u^{\infty}(\hat{x}, \hat{\theta})=-\int_{\partial D} \frac{\partial u}{\partial \nu}(y) e^{-i k \hat{x} \cdot y} d s(y), \quad \hat{x} \in \mathbb{S}^{n-1} .
$$




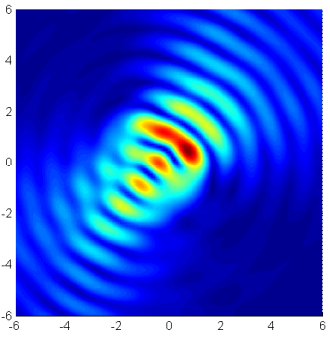

(a) $k=3$

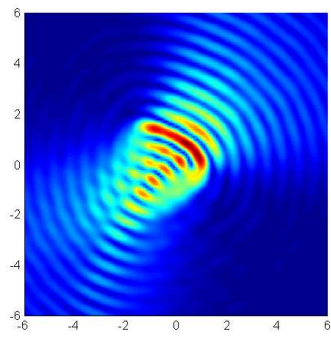

(b) $k=5$

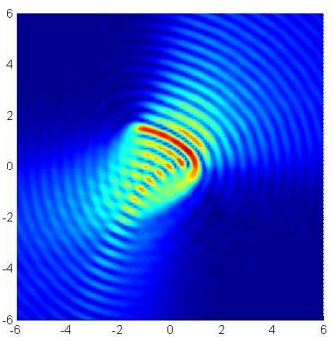

(c) $k=7$

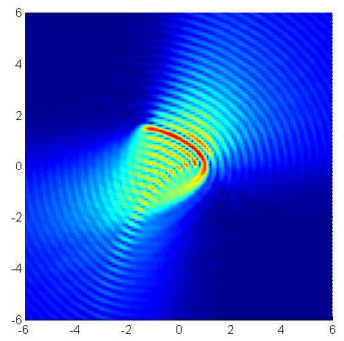

(d) $k=10$

FIGURE 4. Reconstructions of the kite shaped obstacle with observation directions in $(0, \pi / 2), 10 \%$ noise and different wave numbers.

For large wave numbers $k$, i.e., where the Kirchhoff approximation is considered, the far-field pattern is given by [14]

$$
u^{\infty}(\hat{x}, \hat{\theta})=-2 \int_{\partial D_{-}(d)} \frac{\partial e^{i k \hat{\theta} \cdot y}}{\partial \nu(y)} e^{-i k \hat{x} \cdot y} d s(y), \quad \hat{x} \in \mathbb{S}^{n-1},
$$

where $\partial D_{-}(\hat{\theta}):=\{y \in \partial D \mid \nu(y) \cdot \theta<0\}$ is the region illuminated by the plane wave with the incident direction $\hat{\theta}$. Clearly, for a fixed $\hat{\theta} \in \mathbb{S}^{n-1}$, if the illuminated part $\partial D_{-}(\hat{\theta})$ is known in advance or obtained approximately, one may have the far-field data $u^{\infty}(\hat{x}, \hat{\theta})$ for all $\hat{x} \in \mathbb{S}^{n-1}$ by the formula (2.2). Using further the well-known reciprocity relation for the far-field pattern, we have

$$
\begin{aligned}
u^{\infty}(\hat{x}, \hat{\theta}) & =u^{\infty}(-\hat{\theta},-\hat{x}) \\
& =-2 \int_{\partial D_{+}(\hat{x})} \frac{\partial e^{-i k \hat{x} \cdot y}}{\partial \nu(y)} e^{i k \hat{\theta} \cdot y} d s(y), \quad \hat{x} \in \mathbb{S}^{n-1} .
\end{aligned}
$$

The representation (2.3) implies that the shadow domain $\partial D_{-}(\hat{x})$ makes no contribution to the far-field data $u^{\infty}(\hat{x}, \hat{\theta})$. This fact partially explains, in Figures $2-4$, why only the region $\partial D_{+}(\hat{x})$ can be well reconstructed.

We further consider the set $\left\{u^{\infty}(\hat{x}, \hat{\theta}): \hat{x} \in \Gamma, \hat{\theta} \in \Sigma\right\}$ of far-field patterns as data for the inverse problem (1.3), i.e., a limited observation aperture $\Gamma \subset \mathbb{S}^{1}$ and a limited incident aperture $\Sigma \subset \mathbb{S}^{1}$. Of particular interest is the case, where $\Gamma=-\Sigma$, i.e., "backscattering" data is considered. Figure 5 shows the corresponding reconstructions for a sound-soft round square. It is clear that, similar to the previous examples, only the region $\partial D_{+}(\hat{x})$ can be well captured and the quality of the reconstruction deteriorates as the aperture decreases. The numerical examples above show the intrinsic ill-posedness of the inverse problem (1.3) with limited aperture data due to the lack of information. It is remarked that due to the localized feature of the reconstruction method (2.1), if full aperture data is available, say e.g., in the numerical examples in Figure 5, one can clearly recover the full obstacle in an accurate manner. We would also like to emphasize that the imaging functional (2.1) does not work for the phaseless inverse problem (1.4). That is, if only phaseless measurement data is available, even with full aperture, one will encounter even more severe ill-posedness due to the intrinsic lack of information. Clearly, this intrinsic lack of information cannot be remedied by any mathematical tricks unless more a-priori information is complemented. This 


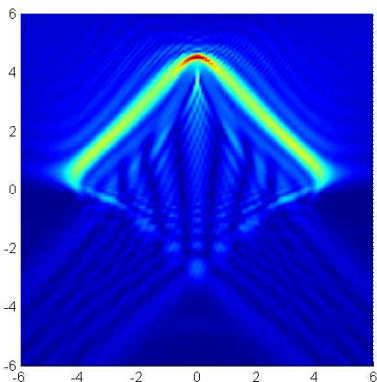

(a) Backscattering data for $\phi \in(\mathrm{b})$ Backscattering data for $\phi \in$ $(0, \pi)$

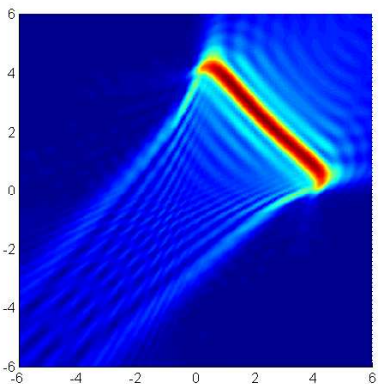

$(0, \pi / 2)$

FigURE 5. Reconstructions of a sound-soft round square by direct sampling method with limited aperture backscattering data, $k=20,10 \%$ noise is added.

naturally leads us to integrate the machine learning techniques into the inverse scattering problems associated with incomplete measurement data. In the next section, we shall briefly go through the main ingredients of the CNN for our subsequent use.

\section{Artificial NeURAL NETWORK}

The concept of artificial neural network (ANN) was proposed by McCulloch and Pitts in 1943, and they also proposed network structure and mathematical descriptions of neurons through the M-P model. In 1986, Rumelhart et. al. proposed the back propagation (BP) algorithm for multi-layer perceptron and used the sigmoid function as the nonlinear mapping [55]. This method solved the nonlinear classification problem effectively, which led to a second wave of neural networks development. In 1988, LeCun et al. began to study convolutional neural networks (CNN) and developed well-known LeNet5 [36], which is a special ANN with convolutional kernel. It is pointed out that the BP algorithm has a gradient disappearance problem which results in very poor learning efficiency. In 2006, Hinton proposed a solution to the gradient disappearance problem in deep network training, that is, unsupervised pre-training initializes weights plus supervised training fine-tuning [25, 26], which led to a third wave of neural networks development. A deep neural network (DNN) is an ANN with multiple layers between the input and output layers, which is based on a hierarchy of composition of linear functions and a given nonlinear activation function. It became popular and widely accepted around 2010 due to the development of efficient learning algorithms [25, 26, 35, 37, 38] and hardware speed-ups such as the use of GPUs. In 2017, AlphaGo defeated Go world champion Ke Jie by using Monte Carlo tree search combined with two deep neural networks. These developments and applications show that artificial neural networks have become very powerful tools in machine learning or artificial intelligence such as image processing and natural language processing [23,61].

Mathematical analysis of neural networks has been developed by several researchers. In 1989, Cybenko and Hornik have shown the "universal approximation theorem" independently, that is, under mild assumptions on the activation function, a feed-forward network with a single hidden layer can approximate continuous functions on compact subsets of $\mathbb{R}^{n}[17,28]$. However, this theorem does not give the relationship between the convergence and the number 
of hidden layers and units, and we refer to some extensions of the universal approximation property [20,21,39]. On the other hand, Barron has analyzed the two-layer neural networks in the Barron spaces around 1993, which is an extension of the Fourier analysis of two-layer sigmoidal neural networks [8,9]. In [56], Shaham et. al. constructed a wavelet frame for approximating functions on smooth manifolds, where the wavelets are computed from Rectified Linear Units (ReLU). Under this construction, they have shown how the specified size depends on the complexity of the function and the curvature of the manifold, which can specify the network architecture to obtain desired approximation properties. Based on [8,9], $\mathrm{E}, \mathrm{Ma}$, and $\mathrm{Wu}$ have establish direct and inverse approximation theorems, for functions in the Barron space for two-layer networks, and also for functions in compositional function space for residual neural network models [19].

With the fast development of optimization algorithms and hardware configuration, the zoo of neural network types grows exponentially, which includes CNN, recurrent neural network (RNN, [55]), long short-term memory (LSTM, [27]), DNN, and etc. Among these different types of neural networks, CNNs are used heavily in image processing. Firstly, the CNNs provide an advantage over feed-forward networks because they are capable of considering locality of features and extracting spatial features. The convolution layer will transform an input into a stack of feature mappings. The depth of the feature map stack depends on how many filters are defined for a layer. Secondly, with the fixed small window size of convolutions, a CNN minimizes the computation sharply compared to a regular neural network.

Next, we present the concepts and notations of the CNN. A CNN consists of $L+1$ layers, where the layer 0 is the input layer, the layers $0<l<L$ are the hidden layers, and the layer $L$ is the output layer. We can adopt the $\mathbb{F}_{12}$ and $\mathbb{F}_{\text {full }}$ defined in what follows in (4.1) as input and output layer respectively to retrieval the full-aperture far-field directly. The activation functions in the hidden layers can be any activation function such as sigmoids, rectified linear units, or hyperbolic tangents. Here, we will use parametric rectified linear unit (PReLU) in the hidden layers.

Parametric Rectified Linear Unit (PReLU) is a type of leaky Rectified Linear Unit (ReLU), where instead of having a predetermined slope such as 0.01 . The slope is a parameter to be determined by the neural network

$$
\sigma(\alpha, x)= \begin{cases}\alpha x, & x<0 \\ x, & x \geq 0\end{cases}
$$

where $\alpha$ is a learned array with the same dimension as $x$.

Convolution layer is composed of several convolution kernels which are used to compute different feature maps. The complete feature maps are obtained by using several different kernels. Let $\mathbf{z}_{i, j}^{l}$ be the input patch centered at location $(i, j)$ of the $l$-th layer and $y_{i, j, k}^{l}$ be the feature value at location $(i, j)$ in the $k$-th feature map of the $l$-th layer, respectively. Then

$$
y_{i, j, k}^{l}=\left(\mathbf{w}_{k}^{l}\right)^{T} \mathbf{z}_{i, j}^{l}+b_{k}^{l},
$$

where $\mathbf{w}_{k}^{l} \in \mathbb{R}^{M \times M}$ and $b_{k}^{l}$ are the weight vector with window size $M \times M$ and bias term of the $k$-th filter of the $l$-th layer, respectively. Note that the kernel $\mathbf{w}_{k}^{l}$, which generates the feature map $y_{:, ., k}^{l}$, is shared. Such a weight sharing mechanism has several advantages. For example, it can reduce the model complexity and make the network easier to train. The activation function in layer $l$ will be denoted by $\sigma^{l}=\sigma(\alpha, x)$. Then the activation value $z_{i, j, k}^{l}$ 
of convolutional feature $y_{i, j, k}^{l}$ can be computed as

$$
z_{i, j, k}^{l}=\sigma^{l}\left(y_{i, j, k}^{l}\right)
$$

Denote $\mathbf{w}=\left\{\mathbf{w}_{k}^{l}\right\}$ and $b=\left\{b_{k}^{l}\right\}$. The schematic representation of general convolution neural network is shown in Fig 6.

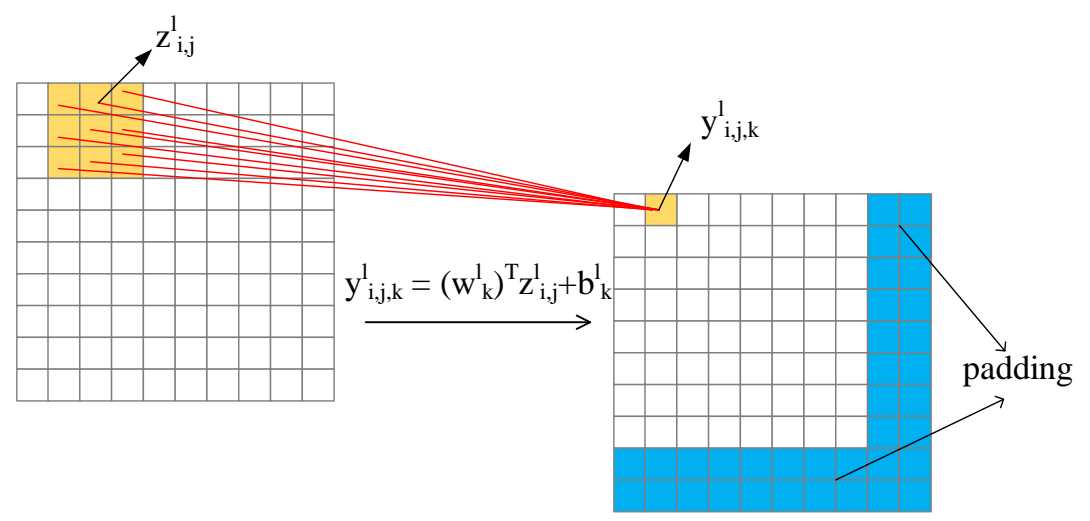

FIGURE 6. Schematic representation of general convolution neural network.

\section{Algorithmic DeVelopment And numerical EXPERIMEnts}

In this section, we present our data retrieval method based on $\mathrm{CNN}$ for the inverse scattering problems (1.3) and (1.4). The proposed method shall first try to recover the full data by using the provided incomplete data via certain training mechanisms through proper CNNs. After the recovery of the full data, we then make use of the imaging method (2.1) to reconstruct the unknown obstacle. We would like to remark that this data retrieval and inverse scattering recovery process can obviously be extended to the other inverse scattering problems with incomplete data. Moreover, mainly due to limiting computing resources, we only consider $2 \mathrm{D}$ examples in what follows, and the extension to three dimensions are obvious if more computing resources are available.

We introduce some general ingredients for our subsequent algorithmic developments through concrete examples. Denote by $\mathcal{X}$ the set of far-field patterns on $\Gamma \times \Sigma$ and $\mathcal{Y}$ the set of far-field patterns on $\mathbb{S}^{1} \times \mathbb{S}^{1}$. Then each limited-aperture far-field pattern $X \in \mathcal{X}$ is mapped to the unique full-aperture far-field pattern

$$
Y=\mathscr{A}(X) \in \mathcal{Y}
$$

where $\mathscr{A}$ denotes the analytic continuation. Our objective is to retrieve the function $\mathscr{A}$ : $\mathcal{X} \rightarrow \mathcal{Y}$ from the samples

$$
\mathcal{S}=\left\{\left(X^{n} \in \mathcal{X}, Y^{n} \in \mathcal{Y}\right)\right\}_{n=1}^{n^{*}},
$$

where $X^{n}, Y^{n}$ are a limited-aperture and the full-aperture far-field data corresponding to the same obstacle $\Omega_{n}$ from the sample set

$$
\mathcal{D}=\left\{\Omega_{n}\right\}_{n=1}^{n^{*}}
$$


i.e.,

$$
\begin{array}{ll}
X^{n}=u^{\infty}\left(\Omega_{n} ; \hat{x}, \hat{\theta}\right), & (\hat{x}, \hat{\theta}) \in \Gamma \times \Sigma, \\
Y^{n}=u^{\infty}\left(\Omega_{n} ; \hat{x}, \hat{\theta}\right), \quad(\hat{x}, \hat{\theta}) \in \mathbb{S}^{1} \times \mathbb{S}^{1} .
\end{array}
$$

Here, $n^{*}$ denotes the total number of samples.

It is generally an open problem as how to formulate and generate a set of random and arbitrary non-selfintersecting shapes. In this paper, we restrict ourself to star shaped domains whose boundaries are defined by the parametric curves

$$
x(t)=r(t) \cos (t), \quad y(t)=r(t) \sin (t), \quad t \in[0,2 \pi] .
$$

We then represent the radius function $r(t)$ by the truncated Fourier series expansion

$$
r(t)=a_{0}\left\{1+\frac{1}{2 N} \sum_{n=1}^{N} n^{-q}\left[a_{n} \cos (n t)+b_{n} \sin (n t)\right]\right\},
$$

where $N \in \mathbb{N}$ is the cut-off frequency, and $a_{n}, b_{n}, n=1, \cdots, N$ are random numbers draw from the uniform distribution in $[-1,1]$. The factor $\frac{1}{2 N}$ is to guarantee $r(t)$ does not change sign so that the resultant shape is non-selfintersecting. The parameter $q$ can be adjusted to control the decreasing rate of the Fourier coefficients and thus the level of smootheness of the shape. The parameter $a_{0}$ can be taken as a fixed value or a random variable.

The range of the sample set can be adjusted by the parameter $N$ and the range of $a_{0}$. A broader range of the sample set implies a broader applicability of the training results, but also requires a larger training set and training time. In the subsequent numerical experiments, we take $N=5, q=0$, and $a_{0}$ to be a random number drawn from the uniform distribution in $[0.5,1.5]$.

We also need to introduce the multi-static response (MSR) matrix and the related properties. The multi-static response matrix $\mathbb{F}_{\text {full }} \in C^{2 m \times 2 m}$ corresponding to the full-aperture far-field data is defined as

$$
\mathbb{F}_{\text {full }}=\left(\begin{array}{cccc}
u_{1,1}^{\infty} & u_{1,2}^{\infty} & \cdots & u_{1,2 m}^{\infty} \\
u_{2,1}^{\infty} & u_{2,2}^{\infty} & \cdots & u_{2,2 m}^{\infty} \\
\vdots & \vdots & \ddots & \vdots \\
u_{2 m, 1}^{\infty} & u_{2 m, 2}^{\infty} & \cdots & u_{2 m, 2 m}^{\infty}
\end{array}\right)
$$

where $u_{i, j}^{\infty}=u^{\infty}\left(\hat{x}_{j} ; \hat{\theta}^{i}\right)$ for $1 \leq i, j \leq 2 m$ corresponding to $2 m$ observation directions $\hat{x}_{j}$ and $2 m$ incident directions $\hat{\theta}^{i}$. Note that $\mathbb{F}_{\text {full }}$ is neither symmetric nor Hermitian, i.e.,

$$
\mathbb{F}_{\text {full }} \neq \mathbb{F}_{\text {full }}^{T} \text { and } \quad \mathbb{F}_{\text {full }} \neq \mathbb{F}_{\text {full }}^{*} \text {. }
$$

Here and throughout the paper we use the superscript " $T$ " and "**" to denote the transpose and the conjugate transpose, respectively, of a matrix.

Generally speaking, we can partition the $2 m$-by- $2 m$ MSR matrix $\mathbb{F}_{\text {full }}$ into a 2 -by-2 block matrix

$$
\mathbb{F}_{\text {full }}=\left(\begin{array}{ll}
\mathbb{F}_{11} & \mathbb{F}_{12} \\
\mathbb{F}_{21} & \mathbb{F}_{22}
\end{array}\right),
$$

where $\mathbb{F}_{11} \in C^{m_{1} \times m_{1}}, \mathbb{F}_{12} \in C^{m_{1} \times m_{2}}, \mathbb{F}_{11} \in C^{m_{2} \times m_{1}}, \mathbb{F}_{12} \in C^{m_{2} \times m_{2}}$, and $m_{1}+m_{2}=2 m$. Here, $\mathbb{F}_{12}$ denotes the limited-aperture far-field data. 
Let $n_{1}$ and $n_{2}$ be the size of train set and test set, respectively. In the sequel, we denote $\mathbb{F}_{\text {full }}^{n}$ by the $n$-th sample in the sample set, and similar notations for sub-block matrices $\mathbb{F}_{i j}^{n}$, $i, j=1,2$.

We proceed to the algorithmic development through four concrete examples.

Example 1. In this example, we consider the standard loss function with the total number of samples $n^{*}=20000$.

Following [58], the architecture of our neural network is a feed-forward stack of five sequential combinations of the convolution, batch normalization and PReLU layers, followed by one fully connected layer. The numbers of filters in the five convolutional layers are 128, 64, 128, 64 and 1, respectively, which are shown in Fig 7. The corresponding window sizes of convolutions are $3 \times 3,2 \times 2,4 \times 4,5 \times 5$, and $4 \times 4$, respectively(i.e. $M=3,2,4,5$, and 4 , respectively for each layer). The stride of the convolution is one, and zero-padding is used. The initial value of the bias is zero. The weight initialization is via the Glorot uniform initializer [22].

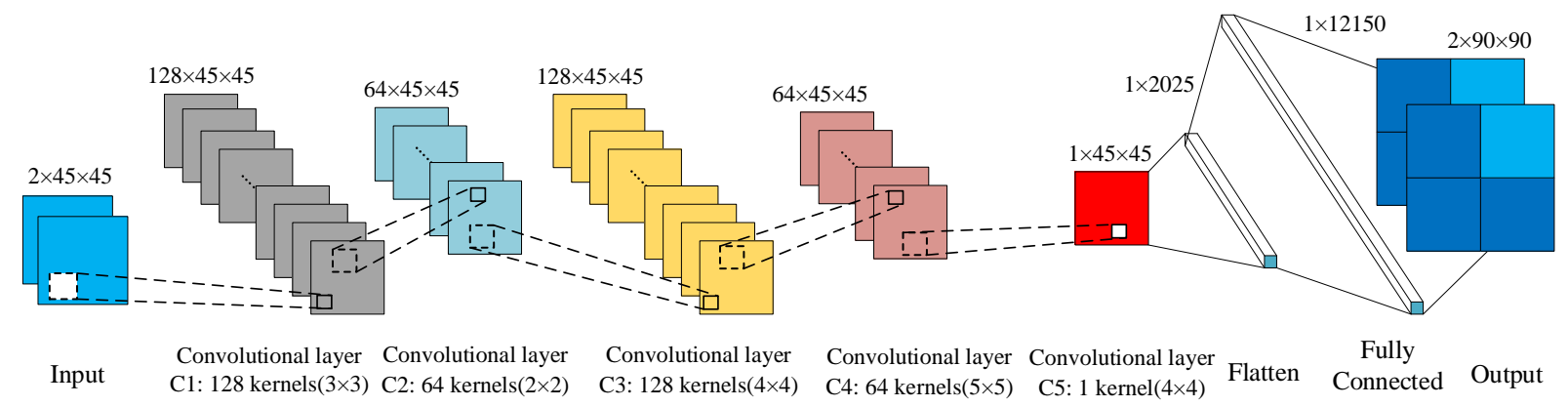

FIGURE 7. The architecture of our convolution neural network in Example 1 and 2.

With this architecture, we train the network with the Adam optimizer, using a mini-batch of 128 samples in each iteration, and 200 epochs. The initial learning rate and forgetting rate of the Adam are the same as in [32]. The total number of samples is $n^{*}=20000$, where the size of the train set is $n_{1}=16000$ and the size of the test set is $n_{2}=4000$. For each sample in the training set $\left(n=1, \cdots, n_{1}\right)$, the input data is $\mathbb{F}_{12}^{n} \in C^{m_{1} \times m_{1}}$ (with $m_{1}=45$ in first three simulations) which is the upper-right quarter of $\mathbb{F}_{\text {full }}^{n}$, while the target data are $\mathbb{F}_{11}^{n}, \mathbb{F}_{12}^{n}, \mathbb{F}_{21}^{n}$, and $\mathbb{F}_{22}^{n}$. That is, our CNN defines a mapping $\mathbb{R}^{m_{1} \times m_{1} \times 2} \rightarrow \mathbb{R}^{2 m \times 2 m \times 2}$ (each complex number has a real part and an imaginary part).

Given the input data $x=\mathbb{F}_{12}^{n} \in \mathbb{R}^{m_{1} \times m_{1} \times 2}$ and the target outputs $y=\left[\mathbb{F}_{11}^{n}, \mathbb{F}_{12}^{n} ; \mathbb{F}_{21}^{n}, \mathbb{F}_{22}^{n}\right] \in$ $\mathbb{R}^{2 m \times 2 m \times 2}$ in the training set, we wish to choose our weights and biases such that $y^{C}(x ; \mathbf{w}, b)$ is a good approximation of $y(x)$, where $y^{C}(x ; \mathbf{w}, b)=\left[\mathbb{F}_{11}^{n, C}, \mathbb{F}_{12}^{n, C} ; \mathbb{F}_{21}^{n, C}, \mathbb{F}_{22}^{n, C}\right]$ denotes the outputs of CNN.

To find the weights and biases, we define the following loss function

$$
\mathcal{L}_{1}=\mathcal{L}_{1}\left(y, y^{C}\right)=\frac{1}{n_{1}} \sum_{n=1}^{n_{1}} \sum_{i, j=1}^{2}\left\|\mathbb{F}_{i j}^{n}-\mathbb{F}_{i j}^{n, C}\right\|_{F}^{2},
$$

where $\|\cdot\|_{F}$ is the Frobenius norm of a matrix. We use the CNN to compute

$$
\mathbf{w}^{*}, b^{*}=\arg \min _{\mathbf{w}, b} \mathcal{L}_{1}\left(y, y^{C}\right)
$$


with the Adam optimizer. We define the following sub-block relative errors

$$
e_{11}^{n}=\frac{\left\|\mathbb{F}_{11}^{n}-\mathbb{F}_{11}^{n, C}\right\|_{F}}{\left\|\mathbb{F}_{11}^{n}\right\|_{F}}, e_{21}^{n}=\frac{\left\|\mathbb{F}_{21}^{n}-\mathbb{F}_{21}^{n, C}\right\|_{F}}{\left\|\mathbb{F}_{21}^{n}\right\|_{F}}, e_{22}^{n}=\frac{\left\|\mathbb{F}_{22}^{n}-\mathbb{F}_{22}^{n, C}\right\|_{F}}{\left\|\mathbb{F}_{22}^{n}\right\|_{F}}, \quad n=n_{1}+1, \cdots, n^{*}
$$

for each sample in the test set, and use the sub-block relative errors of the test set

$$
\bar{e}_{11}=\frac{1}{n_{2}} \sum_{n=n_{1}+1}^{n^{*}} e_{11}^{n}, \bar{e}_{21}=\frac{1}{n_{2}} \sum_{n=n_{1}+1}^{n^{*}} e_{21}^{n}, \bar{e}_{22}=\frac{1}{n_{2}} \sum_{n=n_{1}+1}^{n^{*}} e_{22}^{n},
$$

to check the efficiency of our CNN. Table 1 shows that the sub-block relative errors of the test set are decreasing as $n_{1}$ and $n_{2}$ increase. We emphasize that the errors $\bar{e}_{11}$ and $\bar{e}_{22}$ are smaller than $\bar{e}_{21}$, which is reasonable since $\mathbb{F}_{11}$ and $\mathbb{F}_{22}$ are closer to the $\mathbb{F}_{12}$.

TABLE 1. The sub-block relative errors of test set in Example 1.

\begin{tabular}{|r||c|c|c|c|c|c|c|c|c|}
\hline \multicolumn{1}{|c||}{} & \multicolumn{3}{c|}{ error real(\%) } & \multicolumn{3}{c|}{ error image(\%) } & \multicolumn{3}{c|}{ error norm(\%) } \\
\hline$\left(n_{1}, n_{2}\right)$ & $\bar{e}_{11}$ & $\bar{e}_{21}$ & $\bar{e}_{22}$ & $\bar{e}_{11}$ & $\bar{e}_{21}$ & $\bar{e}_{22}$ & $\bar{e}_{11}$ & $\bar{e}_{21}$ & $\bar{e}_{22}$ \\
\hline \hline$(2000,500)$ & 26.01 & 84.15 & 26.01 & 30.72 & 86.03 & 30.71 & 28.06 & 84.99 & 30.71 \\
\hline$(4000,1000)$ & 19.01 & 67.55 & 19.00 & 22.49 & 68.43 & 22.49 & 20.52 & 67.92 & 20.52 \\
\hline$(8000,2000)$ & 14.22 & 51.15 & 14.22 & 16.88 & 52.12 & 16.88 & 15.38 & 51.54 & 15.38 \\
\hline$(16000,4000)$ & 10.66 & 38.88 & 10.66 & 12.61 & 39.82 & 12.61 & 11.50 & 39.27 & 11.50 \\
\hline
\end{tabular}

For $n=n_{1}+1, \cdots, n^{*}$, combining the outputs $\left[\mathbb{F}_{11}^{n, C}, \mathbb{F}_{21}^{n, C}, \mathbb{F}_{22}^{n, C}\right]$ and the input $\mathbb{F}_{12}^{n}$ of CNN, we can obtain the recovery multi-static response matrix as

$$
\widetilde{\mathbb{F}}_{\text {full }}^{n}=\left(\begin{array}{cc}
\mathbb{F}_{11}^{n, C} & \mathbb{F}_{12}^{n} \\
\mathbb{F}_{21}^{n, C} & \mathbb{F}_{22}^{n, C}
\end{array}\right), \quad n=n_{1}+1, \cdots, n^{*},
$$

for each sample in the test set. Given a noise-free $2 m \times 2 m$ sample $\mathbb{F}_{\text {full }}^{n}$ in the test set and its approximation $\widetilde{\mathbb{F}}_{f u l l}^{n}$, define

$$
\begin{aligned}
e^{n} & =\frac{\left\|\mathbb{F}_{11}^{n}-\mathbb{F}_{11}^{n, C}\right\|_{F}+\left\|\mathbb{F}_{21}^{n}-\mathbb{F}_{21}^{n, C}\right\|_{F}+\left\|\mathbb{F}_{22}^{n}-\mathbb{F}_{22}^{n, C}\right\|_{F}}{\left\|\mathbb{F}_{11}^{n}\right\|_{F}+\left\|\mathbb{F}_{21}^{n}\right\|_{F}+\left\|\mathbb{F}_{22}^{n}\right\|_{F}}, \\
\operatorname{MSE}^{n} & =\frac{1}{2 m \cdot 2 m} \sum_{i=1}^{2 m} \sum_{j=1}^{2 m}\left|\mathbb{F}_{\text {full }}^{n}(i, j)-\widetilde{\mathbb{F}}_{\text {full }}^{n}(i, j)\right|^{2}, \\
\mathrm{PSNR}^{n} & =10 \cdot \log _{10}\left(\frac{\max _{i, j}\left|\mathbb{F}_{\text {full }}^{n}(i, j)\right|}{\operatorname{MSE}^{n}}\right) .
\end{aligned}
$$

In order to check the efficiency of our $\mathrm{CNN}$, we also introduce the relative error, mean square error (MSE), and peak signal-to-noise ratio (PSNR) of the test set as follows:

$$
\bar{e}=\frac{1}{n_{2}} \sum_{n=n_{1}+1}^{n^{*}} e^{n}, \overline{\mathrm{MSE}}=\frac{1}{n_{2}} \sum_{n=n_{1}+1}^{n^{*}} \mathrm{MSE}^{n}, \overline{\mathrm{PSNR}}=\frac{1}{n_{2}} \sum_{n=n_{1}+1}^{n^{*}} \operatorname{PSNR}^{n} .
$$

Table 2 shows that the relative errors and $\overline{\mathrm{MSE}}$ s of the test set are decreasing as $n_{1}$ and $n_{2}$ increase. Meanwhile, the $\overline{\mathrm{PSNR}} \mathrm{s}$ of test set are increasing as $n_{1}$ and $n_{2}$ increase, which means the more samples in the train test, the better the prediction will be. 
TABLE 2. The relative errors, $\overline{\mathrm{MSE}} \mathrm{s}$, and $\overline{\mathrm{PSNR}} \mathrm{s}$ of test set in Example 1.

\begin{tabular}{|r||c|c|c|c|c|c|c|c|c|}
\hline \multicolumn{1}{|c||}{} & \multicolumn{3}{|c|}{ relative error $\bar{e}(\%)$} & \multicolumn{3}{c|}{$\overline{\mathrm{MSE}}\left(10^{-2}\right)$} & \multicolumn{3}{c|}{$\overline{\overline{\text { PSNR }}}$} \\
\hline$\left(n_{1}, n_{2}\right)$ & real & image & norm & real & image & norm & real & image & norm \\
\hline \hline$(2000,500)$ & 39.13 & 44.03 & 41.40 & 9.372 & 9.384 & 5.430 & 19.21 & 16.22 & 22.37 \\
\hline$(4000,1000)$ & 34.06 & 38.47 & 36.11 & 6.979 & 7.042 & 4.371 & 20.28 & 17.27 & 22.24 \\
\hline$(8000,2000)$ & 23.97 & 27.09 & 25.41 & 3.445 & 3.440 & 2.594 & 23.24 & 20.22 & 25.65 \\
\hline$(16000,4000)$ & 16.63 & 18.80 & 17.63 & 1.691 & 1.692 & 1.365 & 26.46 & 23.46 & 28.63 \\
\hline
\end{tabular}

Fig 8 shows the numerical constructions of the 1st shape from the test set by the full MSR matrix $\mathbb{F}_{\text {full }}^{n}$, a limited MSR matrix $\mathbb{F}_{12}^{n}$, and the recovery MSR matrix $\widetilde{\mathbb{F}}_{\text {full }}^{n}$ via CNN. Although the average error $\bar{e}_{21}$ is around $20 \%$, the numerical construction by the recovery MSR matrix via CNN is indistinguishable from the numerical construction by the full MSR matrix.
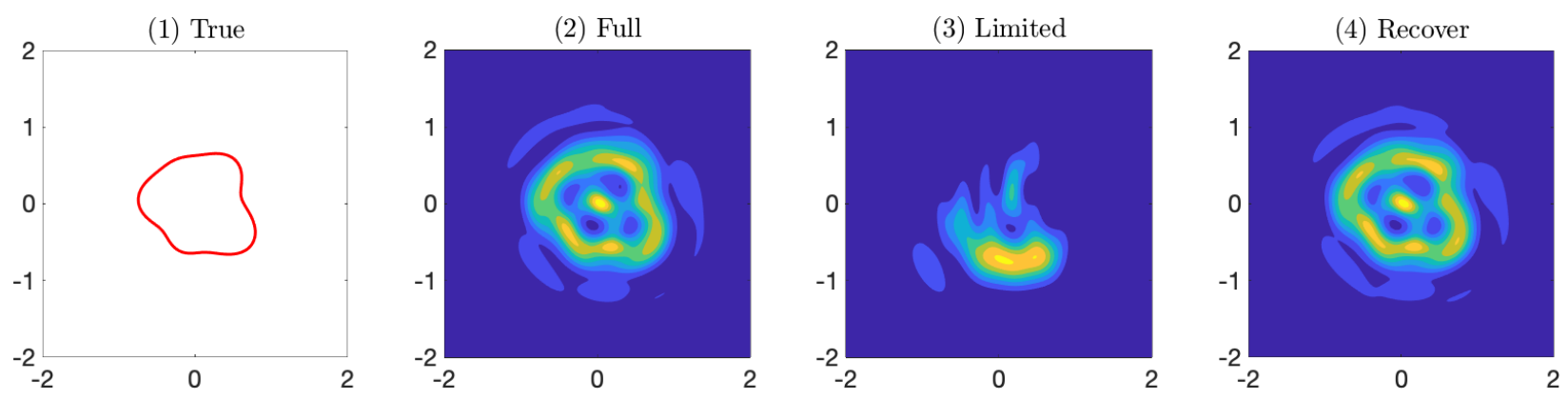

Figure 8. Numerical constructions of the 1st shape in the test set. (1) the true shape; (2) reconstructed shape from the full MSR matrix $\mathbb{F}_{\text {full }}^{n}$; (3) reconstructed shape from the limited MSR matrix $\mathbb{F}_{12}^{n}$; (4) reconstructed shape from the recovered MSR matrix $\widetilde{\mathbb{F}}_{\text {full }}^{n}$ via CNN.

Example 2. In this example, we consider the loss function with a regularization term. The architecture of convolution neural network, the input data $x=\mathbb{F}_{12}^{n} \in \mathbb{R}^{m_{1} \times m_{1} \times 2}$, and the target outputs $y=\left[\mathbb{F}_{11}^{n}, \mathbb{F}_{12}^{n} ; \mathbb{F}_{21}^{n}, \mathbb{F}_{22}^{n}\right] \in \mathbb{R}^{m \times m \times 2}$ are the same as in Example 1 . The total number of samples is $n^{*}=50000$, where the size of the training set is $n_{1}=40000$ and the size of the test set is $n_{2}=10000$.

Define the modified loss function as

$$
\begin{aligned}
& \mathcal{L}_{2}\left(y, y^{C}\right)=\mathcal{L}_{1}\left(y, y^{C}\right) \\
& +\frac{\alpha}{n_{1}} \sum_{n=1}^{n_{1}}\left\{\left|\mathbb{F}_{11}^{n, C} \cap \mathbb{F}_{12}^{n}\right|_{H^{1}}^{2}+\left|\mathbb{F}_{21}^{n, C} \cap \mathbb{F}_{22}^{n, C}\right|_{H^{1}}^{2}+\left|\mathbb{F}_{11}^{n, C} \cap \mathbb{F}_{21}^{n}\right|_{H^{1}}^{2}+\left|\mathbb{F}_{12}^{n} \cap \mathbb{F}_{22}^{n}\right|_{H^{1}}^{2}\right\},
\end{aligned}
$$

where $\mathcal{L}_{1}$ is specified in (4.2), the second summation is the regularization term on the interfaces of each sub-block matrix, and the regularization parameter $\alpha=10^{-3}$.

Table 3 and Table 4 show that the relative errors and $\overline{M S E}$ s of the test set are decreasing as $n_{1}$ and $n_{2}$ increase, while the $\overline{\mathrm{PSNR}}$ s of the test set are increasing as $n_{1}$ and $n_{2}$ increase. Meanwhile the the relative errors and MSEs of $\mathcal{L}_{2}$ are smaller than the $\mathcal{L}_{1}$, and $\overline{\mathrm{PSNR}} \mathrm{s}$ of 
$\mathcal{L}_{2}$ are great than the $\mathcal{L}_{1}$. These mean the regularization term reduces the prediction error efficiently.

TABLE 3. The relative errors, $\overline{\mathrm{MSE}} \mathrm{s}$, and $\overline{\mathrm{PSNR}} \mathrm{s}$ of test set in Example 2 without regularization term.

\begin{tabular}{|r||c|c|c|c|c|c|c|c|c|}
\hline \multicolumn{1}{|c||}{} & \multicolumn{2}{|c|}{ relative error $\bar{e}(\%)$} & \multicolumn{3}{c|}{$\overline{\mathrm{MSE}}\left(10^{-2}\right)$} & \multicolumn{3}{c|}{$\overline{\text { PSNR }}$} \\
\hline$\left(n_{1}, n_{2}\right)$ & real & image & norm & real & image & norm & real & image & norm \\
\hline \hline$(5000,1250)$ & 29.91 & 33.58 & 31.62 & 5.489 & 5.511 & 3.650 & 21.51 & 18.59 & 24.31 \\
\hline$(10000,2500)$ & 21.54 & 24.32 & 22.83 & 2.878 & 2.894 & 2.130 & 24.33 & 21.31 & 26.62 \\
\hline$(20000,5000)$ & 15.67 & 17.74 & 16.63 & 1.438 & 1.439 & 1.198 & 26.85 & 23.84 & 28.95 \\
\hline$(40000,10000)$ & 12.22 & 13.85 & 12.97 & 0.818 & 0.816 & 0.712 & 28.84 & 25.82 & 30.80 \\
\hline
\end{tabular}

TABLE 4. The relative errors, $\overline{\mathrm{MSE}} \mathrm{s}$, and $\overline{\mathrm{PSNR}}$ s of test set in Example 2 with regularization term.

\begin{tabular}{|r||c|c|c|c|c|c|c|c|c|}
\hline \multicolumn{1}{|c||}{} & \multicolumn{2}{|c|}{ relative error $\bar{e}(\%)$} & \multicolumn{3}{c|}{$\overline{\mathrm{MSE}}\left(10^{-2}\right)$} & \multicolumn{3}{c|}{$\overline{\text { PSNR }}$} \\
\hline$\left(n_{1}, n_{2}\right)$ & real & image & norm & real & image & norm & real & image & norm \\
\hline \hline$(5000,1250)$ & 30.08 & 33.63 & 33.73 & 5.372 & 5.319 & 3.686 & 21.34 & 18.47 & 23.91 \\
\hline$(10000,2500)$ & 21.71 & 24.43 & 22.97 & 2.869 & 2.852 & 2.128 & 24.16 & 21.18 & 26.64 \\
\hline$(20000,5000)$ & 15.26 & 17.17 & 16.14 & 1.390 & 1.383 & 1.149 & 27.12 & 24.18 & 29.30 \\
\hline$(40000,10000)$ & 12.00 & 13.45 & 12.67 & 0.798 & 0.786 & 0.677 & 29.02 & 26.10 & 31.20 \\
\hline
\end{tabular}

As an example, let's consider the square shape with corners at $(1.5,0),(0,1.5),(-1.5,0)$ and $(0,-1.5)$. This is a star shape with the radius function given by

$$
r(t)= \begin{cases}1 /(\sin t+\cos t), & t \in\left[0, \frac{\pi}{2}\right], \\ 1 /(\sin t-\cos t), & t \in\left[\frac{\pi}{2}, \pi\right], \\ 1 /(-\sin t-\cos t), & t \in\left[\pi, \frac{3 \pi}{2}\right], \\ 1 /(-\sin t+\cos t), & t \in\left[\frac{3 \pi}{2}, 2 \pi\right] .\end{cases}
$$

One can verify the Fourier coefficients of $r(t)$ lies within the range of possible random numbers for the training and test sets, but it is not one of the 20000 actual samples used in this experiment.

Denote the full MSR matrix $\mathbb{F}_{\text {full }}^{s}$ by

$$
\mathbb{F}_{\text {full }}^{s}=\left(\begin{array}{ll}
\mathbb{F}_{11}^{s} & \mathbb{F}_{12}^{s} \\
\mathbb{F}_{21}^{s} & \mathbb{F}_{22}^{s}
\end{array}\right) .
$$

Let $y^{s, C}=\left[\mathbb{F}_{11}^{s, C}, \mathbb{F}_{12}^{s, C} ; \mathbb{F}_{21}^{s, C}, \mathbb{F}_{22}^{s, C}\right]$ be the predict output of CNN corresponding the input $x^{s}=\mathbb{F}_{12}^{s}$, we can obtain the multi-static response matrix as

$$
\widetilde{\mathbb{F}}_{\text {full }}^{s}=\left(\begin{array}{cc}
\mathbb{F}_{11}^{s, C} & \mathbb{F}_{12}^{s} \\
\mathbb{F}_{21}^{s, C} & \mathbb{F}_{22}^{s, C}
\end{array}\right) .
$$


As a benchmark, we should compare the result with the nearest sample in the training set. Let

$$
s^{*}=\arg \min _{1 \leq n \leq n_{1}}\left\{\left\|\mathbb{F}_{12}^{s}-\mathbb{F}_{12}^{n}\right\|_{F}^{2}\right\}
$$

for phased data, or

$$
s^{*}=\arg \min _{1 \leq n \leq n_{1}}\left\{\left\|\left|\mathbb{F}_{12}^{s}\right|-\left|\mathbb{F}_{12}^{n}\right|\right\|_{F}^{2}\right\}
$$

for phaseless data. Then the nearest sample is given by

$$
\widetilde{\mathbb{F}}_{\text {full }}^{s^{*}}=\left(\begin{array}{cc}
\mathbb{F}_{11}^{s^{*}} & \mathbb{F}_{12}^{s^{*}} \\
\mathbb{F}_{21}^{s^{*}} & \mathbb{F}_{22}^{s^{*}}
\end{array}\right) .
$$

(1) Full

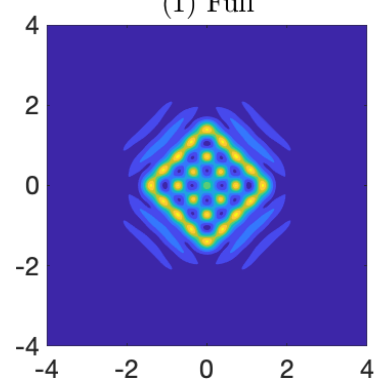

(2) Limited

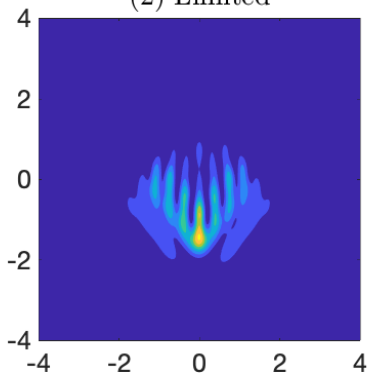

(3) Recover

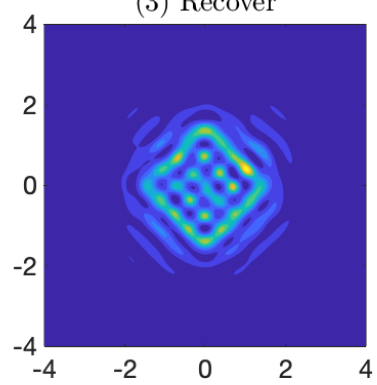

(4) Nearest

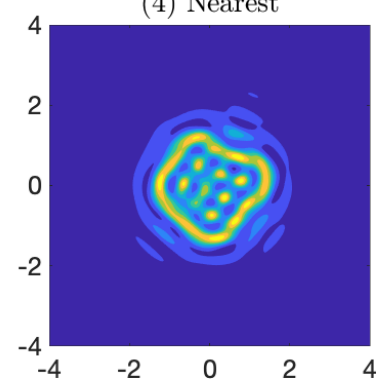

FIGURE 9. Numerical constructions for the square-shaped obstacle by (1) the full MSR matrix $\mathbb{F}_{\text {full }}^{s} ;(2)$ the limited MSR matrix $\mathbb{F}_{12}^{s} ;(3)$ the recovery MSR matrix $\widetilde{\mathbb{F}}_{\text {full }}^{s}$ via $\mathrm{CNN} ;(4)$ the nearest sample MSR matrix $\widetilde{\mathbb{F}}_{\text {full }}^{s^{*}}$.

Fig 9 shows the numerical constructions for square-shaped obstacle by the full MSR matrix $\mathbb{F}_{\text {full }}^{s}$, a limited MSR matrix $\mathbb{F}_{12}^{s}$, the recovery MSR matrix $\widetilde{\mathbb{F}}_{\text {full }}^{s}$ via $\mathrm{CNN}$, and the nearest sample MSR matrix $\widetilde{\mathbb{F}}_{\text {full }}^{s^{*}}$. The corresponding relative errors of recovery MSR matrix $\widetilde{\mathbb{F}}_{\text {full }}^{s}$ and the nearest sample MSR matrix $\widetilde{\mathbb{F}}_{\text {full }}^{s^{*}}$ are listed in Table 5. Although the relative error of the test set $\bar{e}$ is around $13 \%$, the numerical construction by the recovery MSR matrix via CNN is very close to the numerical construction by the full MSR matrix, and much better than the reconstructions from the limited aperture data or the nearest sample.

TABLE 5. The relative errors of recovery MSR matrix $\widetilde{\mathbb{F}}_{\text {full }}^{s}$ and the nearest sample MSR matrix $\widetilde{\mathbb{F}}_{\text {full }}^{s^{*}}$ in Example 2 with regularization term.

\begin{tabular}{|c||c|c|c|}
\hline \multicolumn{1}{|c||}{} & \multicolumn{3}{c|}{ relative error (\%) } \\
\hline matrix type & real & image & norm \\
\hline \hline$\widetilde{\mathbb{F}}_{\text {full }}^{s}$ & 32.00 & 30.42 & 31.25 \\
\hline$\widetilde{\mathbb{F}}_{\text {full }}^{\text {sul }}$ & 66.75 & 69.21 & 67.94 \\
\hline
\end{tabular}

Example 3. In this example, we consider the phaseless obstacle reconstruction problem (1.4). This problem has less information, and thus, is more difficult to reconstruct.

Here, the input data has just module information, that is, $x=\mathbb{F}_{12}^{n} \in \mathbb{R}^{m_{1} \times m_{1}}$, and the target outputs remain unchanged $y=\left[\mathbb{F}_{11}^{n}, \mathbb{F}_{12}^{n} ; \mathbb{F}_{21}^{n}, \mathbb{F}_{22}^{n}\right] \in \mathbb{R}^{2 m \times 2 m \times 2}$. The architecture of 
convolution neural network is similar to previous examples, except that the input layer has only one matrix input (cf. Fig 10). The number of samples is $n^{*}=50000$, while the size of the train set is $n_{1}=40000$ and the size of the test set is $n_{2}=10000$.

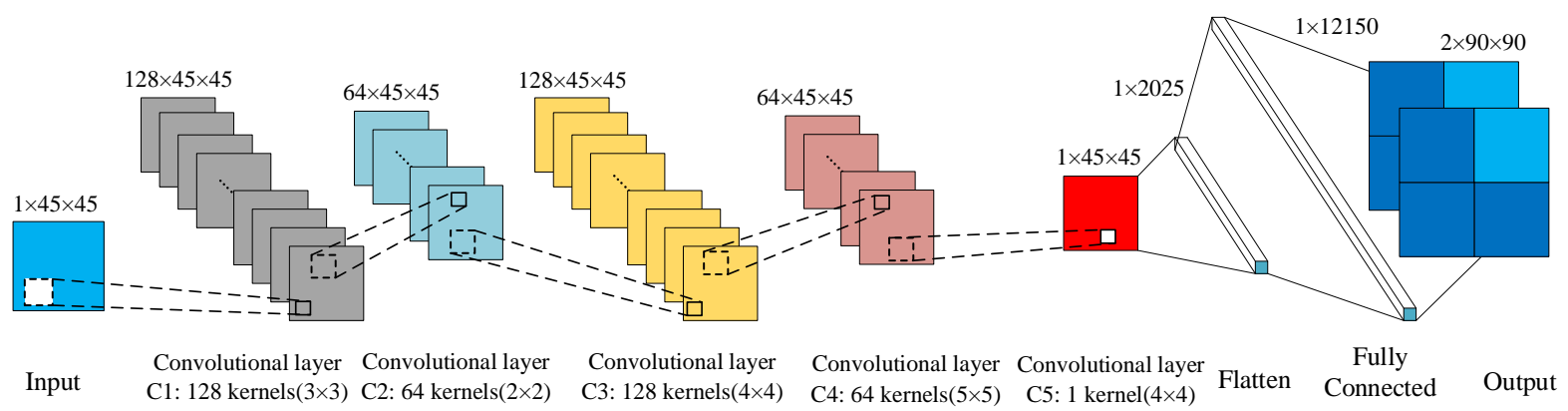

FigurE 10. The architecture of our convolution neural network in Example 3.

We adopt the same loss function $\mathcal{L}_{2}$ as in Example 2. Table 6 shows that the relative errors and $\overline{\mathrm{MSE}}$ s of the test set are decreasing as $n_{1}$ and $n_{2}$ increase, while the $\overline{\text { PSNR }}$ s of the test set are increasing as $n_{1}$ and $n_{2}$ increase. It is easy to see from Table 3 and Table 4 that, the results of phaseless case are worse than that of phase case because we have less information.

TABLE 6 . The relative errors, $\overline{\mathrm{MSE}} \mathrm{s}$, and $\overline{\mathrm{PSNR}}_{\mathrm{S}}$ of the test set in Example 3 with regularization term.

\begin{tabular}{|r||c|c|c|c|c|c|c|c|c|}
\hline \multicolumn{1}{|c||}{} & \multicolumn{2}{|c|}{ relative error $\bar{e}(\%)$} & \multicolumn{3}{c|}{ MSE $\left(10^{-2}\right)$} & \multicolumn{3}{c|}{ PSNR } \\
\hline$\left(n_{1}, n_{2}\right)$ & real & image & norm & real & image & norm & real & image & norm \\
\hline \hline$(5000,1250)$ & 57.49 & 64.95 & 60.91 & 20.535 & 20.615 & 11.332 & 15.87 & 12.84 & 20.24 \\
\hline$(10000,2500)$ & 46.92 & 53.41 & 49.90 & 12.562 & 12.719 & 6.648 & 17.33 & 14.23 & 21.65 \\
\hline$(20000,5000)$ & 41.77 & 47.73 & 44.51 & 9.860 & 10.008 & 5.266 & 18.30 & 15.18 & 22.55 \\
\hline$(40000,10000)$ & 37.97 & 43.28 & 40.40 & 7.510 & 7.573 & 4.408 & 18.91 & 15.81 & 22.91 \\
\hline
\end{tabular}

As a test, we consider the unit circle centered at the origin, whose Fourier coefficients lies within the possible random numbers but not one of the 50000 samples in this example. Let $\mathbb{F}_{\text {full }}^{c}, \mathbb{F}_{12}^{c}, \widetilde{\mathbb{F}}_{\text {full }}^{c}, \widetilde{\mathbb{F}}_{\text {full }}^{c^{*}}$ be the corresponding full MSR matrix, limited MSR matrix, recovery MSR matrix via CNN, and the nearest sample MSR matrix, respectively, in the same manner as in Example 2. Fig 11 shows the numerical constructions of the circle by the full MSR matrix $\mathbb{F}_{\text {full }}^{c}$, a limited MSR matrix $\mathbb{F}_{12}^{c}$, the recovery MSR matrix $\widetilde{\mathbb{F}}_{\text {full }}^{c}$ via $\mathrm{CNN}$, and the nearest sample MSR matrix $\widetilde{\mathbb{F}}_{\text {full }}^{c^{*}}$. Even for the phaseless case, the numerical construction by the recovery MSR matrix via CNN is much better than the reconstruction from the original limited aperture data. The corresponding relative errors of recovery MSR matrix $\widetilde{\mathbb{F}}_{\text {full }}^{c}$ and the nearest sample MSR matrix $\widetilde{\mathbb{F}}_{\text {full }}^{c^{*}}$ are listed in Table 7 . In fact, it is easy to see from Table 7 that the recovered MSR matrix via CNN is slightly better than the nearest sample recovery multi-static response matrix under the $\|\cdot\|_{F}$-norm.

Example 4. In this example, we consider the less information of phase case. The architecture of convolution neural network is similar to the one used in Example 1, except the input data 

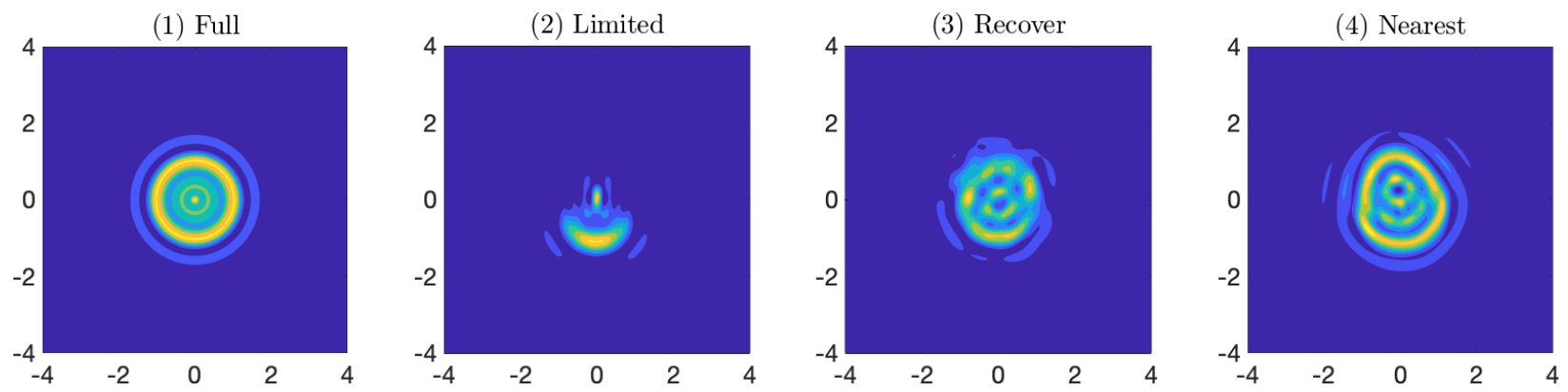

FiguRE 11. Numerical constructions for circle-shape obstacle by (1) the full MSR matrix $\mathbb{F}_{\text {full }}^{c} ;(2)$ the limited MSR matrix $\mathbb{F}_{12}^{c} ;(3)$ the recovery MSR matrix $\widetilde{\mathbb{F}}_{\text {full }}^{c}$ via CNN; (4) nearest sample MSR matrix $\widetilde{\mathbb{F}}_{\text {full }}^{c^{*}}$.

TABLE 7. The relative errors of recovery MSR matrix $\widetilde{\mathbb{F}}_{\text {full }}^{c}$ and the nearest sample MSR matrix $\widetilde{\mathbb{F}}_{\text {full }}^{c^{*}}$ in Example 3 with regularization term.

\begin{tabular}{|c||c|c|c|}
\hline \multicolumn{1}{|c||}{} & \multicolumn{3}{c|}{ relative error (\%) } \\
\hline matrix type & real & image & norm \\
\hline \hline$\widetilde{\mathbb{F}}_{\text {full }}^{s}$ & 56.16 & 57.42 & 56.66 \\
\hline$\widetilde{\mathbb{F}}_{\text {full }}^{s^{*}}$ & 69.05 & 92.99 & 79.50 \\
\hline
\end{tabular}

$x=\mathbb{F}_{12}^{n} \in \mathbb{R}^{m_{1} \times m_{1} \times 2}$ with $m_{1}=30$. The total number of samples is $n^{*}=50000$, while the size of the train set is $n_{1}=40000$ and the size of the test set is $n_{2}=10000$.

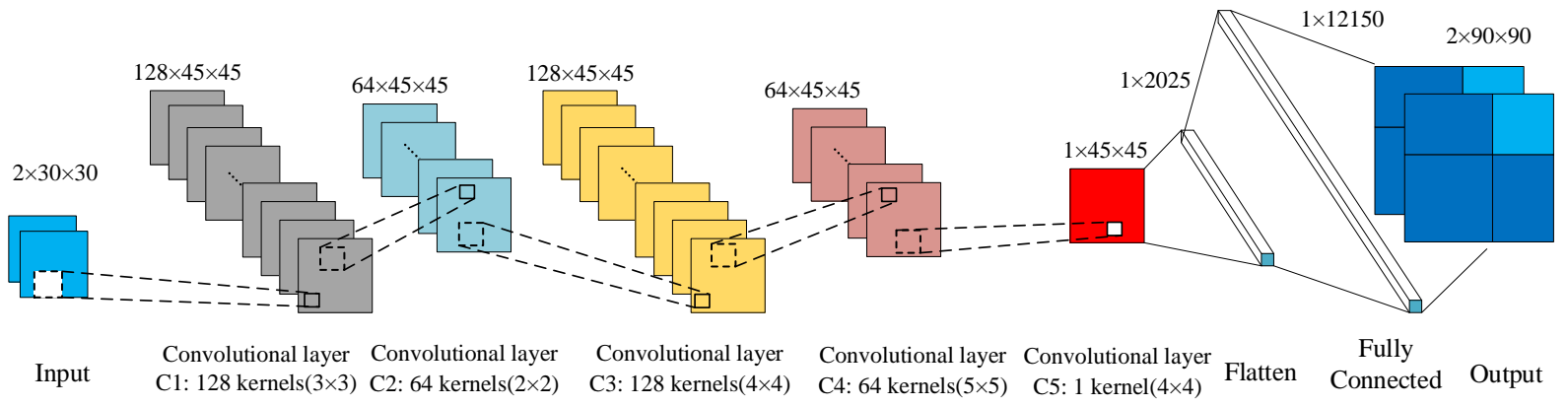

FIGURE 12. The architecture of our convolution neural network in Example 4.

It is easy to see from Table 4 and Table 6 that, the results of $m_{1}=30$ are worse than that of $m_{1}=45$ because that we have less information.

In order to show the robustness of our algorithm, we choose 30 rows and 30 columns randomly in the sub-block $\mathbb{F}_{12} \in C^{m \times m}$ with $m=45$. The architecture of convolution neural network is similar to Fig 12 , except the input data $x=\mathbb{F}_{12}^{n} \in \mathbb{R}^{m_{1} \times m_{1} \times 2}$ with $m_{1}=30$ chosen randomly (see Fig 13).

It is easy to see from Table 4 and Table 8 that, the results of using randomly chosen inputs with $m_{1}=30$ are worse than that of $m_{1}=45$ because we have less information. But the 
TABLE 8. The relative errors, $\overline{\mathrm{MSE}} \mathrm{s}$, and $\overline{\mathrm{PSNR}} \mathrm{s}$ of test set in Example 4 with regularization term and input data $m_{1}=30$.

\begin{tabular}{|r||c|c|c|c|c|c|c|c|c|}
\hline \multicolumn{1}{|c||}{ error } & \multicolumn{3}{|c|}{ relative error $\bar{e}(\%)$} & \multicolumn{3}{c|}{$\overline{\mathrm{MSE}}\left(10^{-2}\right)$} & \multicolumn{3}{c|}{$\overline{\overline{P S N R}}$} \\
\hline$\left(n_{1}, n_{2}\right)$ & real & image & norm & real & image & norm & real & image & norm \\
\hline \hline$(5000,1250)$ & 30.72 & 34.75 & 32.58 & 5.572 & 5.657 & 3.821 & 21.17 & 18.18 & 23.99 \\
\hline$(10000,2500)$ & 24.86 & 28.13 & 26.37 & 3.505 & 3.525 & 2.542 & 22.81 & 19.80 & 25.49 \\
\hline$(20000,5000)$ & 18.93 & 21.37 & 20.06 & 2.116 & 2.128 & 1.652 & 25.25 & 22.27 & 27.54 \\
\hline$(40000,10000)$ & 15.14 & 17.11 & 16.05 & 1.295 & 1.290 & 1.029 & 27.05 & 24.04 & 29.41 \\
\hline
\end{tabular}
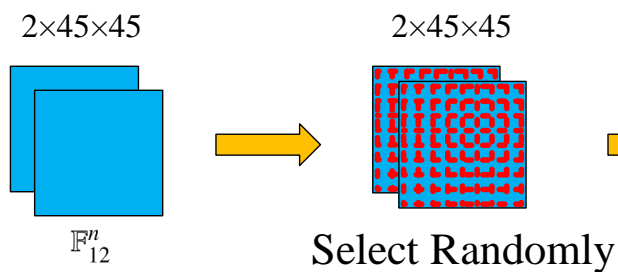

$2 \times 30 \times 30$

FiguRE 13. The architecture of our convolution neural network in Example 4 with input being chosen randomly.

results of $m_{1}=30$ chosen randomly are only slightly different from that of using static inputs as $n_{1}$ and $n_{2}$ increase (Table 8 and Table 9).

TABLE 9. The relative errors, $\overline{\mathrm{MSE}} \mathrm{s}$, and $\overline{\mathrm{PSNR}} \mathrm{s}$ of test set in Example 4 with regularization term and input data $m_{1}=30$ chosen randomly.

\begin{tabular}{|r||c|c|c|c|c|c|c|c|c|}
\hline error & \multicolumn{2}{|c|}{ relative error $\bar{e}(\%)$} & \multicolumn{3}{c|}{$\overline{\mathrm{MSE}}\left(10^{-2}\right)$} & \multicolumn{3}{c|}{$\overline{\mathrm{PSNR}}$} \\
\hline$\left(n_{1}, n_{2}\right)$ & real & image & norm & real & image & norm & real & image & norm \\
\hline \hline$(5000,1250)$ & 33.79 & 37.90 & 35.70 & 6.580 & 6.541 & 4.152 & 20.31 & 17.39 & 23.32 \\
\hline$(10000,2500)$ & 27.44 & 31.22 & 29.20 & 4.295 & 4.371 & 3.020 & 22.01 & 18.95 & 24.67 \\
\hline$(20000,5000)$ & 20.36 & 23.06 & 21.62 & 2.495 & 2.509 & 1.936 & 24.69 & 21.67 & 27.01 \\
\hline$(40000,10000)$ & 15.24 & 17.24 & 16.16 & 1.340 & 1.334 & 1.115 & 27.07 & 24.05 & 29.27 \\
\hline
\end{tabular}

\section{ACKNOWLEDGMENT}

The work of K. Zhang was supported by the NSF of China under the grant No. 11871245, 11771179, and by the Key Laboratory of Symbolic Computation and Knowledge Engineering of Ministry of Education, Jilin University (93K172018Z01). The authors would like to acknowledge the helpful suggestion and discussion with Prof. Hongyu Liu of CityU, Prof. Yuliang Wang of HKBU, and Prof. Xiaodong Liu of AMT.

\section{REFERENCES}

[1] C.Y. Ahn, K. Jeon, Y.K. Ma and W.K. Park, A study on the topological derivative-based imaging of thin electromagnetic inhomogeneities in limited-aperture problems, Inverse Problems 30 (2014), 105004. 
[2] H. Ammari, J. Garnier and K. Solna, Partial data resolving power of conductivity imaging from boundary measurements, SIAM J. Math. Anal. 45 (2013), 1704-1722.

[3] H. Ammari and H. Kang, Reconstruction of small inhomogeneities from boundary measurements, Lecture Notes in Mathematics, 1846, Springer-Verlag, Berlin, 2004.

[4] H. Ammari, E. Iakovleva, D. Lesselier and G. Perrusson, MUSIC-type electromagnetic imaging of a collection of small three-dimensional inclusions, SIAM J. Sci. Comput. 29 (2007), 674-709.

[5] H. Ammari, E. Iakovleva and D. Lesselier, A MUSIC algorithm for locating small inclusions buried in a half-space from the scattering amplitude at a fixed frequency, Multiscale Model. Simul. 3 (2005), $597-628$.

[6] D. Atkinson, Analytic extrapolations and inverse problems, Applied Inverse Problems, pp. 111-121, Berlin, 1978.

[7] G. Bao and J. Liu, Numerical solution of inverse problems with multi-experimental limited aperture data, SIAM J. Sci. Comput. 25 (2003), 1102-1117.

[8] A. R. Barron, Universal approximation bounds for superpositions of a sigmoidal function, Information Theory, IEEE Transactions 39 (1993), 930-945.

[9] A. R. Barron, Approximation and estimation bounds for artificial neural networks, Machine Learning 14 (1994), 115-133.

[10] L. Beilina and M. V. Klibanov, Approximate global convergence in imaging of land mines from backscattered data, Applied Inverse Problems, pp. 15-36, Springer, New York, 2013.

[11] F. Cakoni, D. Colton and H. Haddar, Inverse scattering theory and transmission eigenvalues, in: CBMS-NSF Regional Conference Series in Applied Mathematics, 88, Society for Industrial and Applied Mathematics (SIAM), Philadelphia, PA, 2016.

[12] F. Cakoni, D. Colton and P. Monk, The linear sampling method in inverse electromagnetic scattering, in: CBMS-NSF Regional Conference Series in Applied Mathematics, 80, Society for Industrial and Applied Mathematics (SIAM), Philadelphia, PA, 2011.

[13] D. Colton, J. Coyle and P. Monk, Recent developments in inverse acoustic scattering theory, SIAM Rev. 42 (2000), 369-414.

[14] D. Colton and R. Kress, Inverse Acoustic and Electromagnetic Scattering Theory (Third Edition), Springer, 2013.

[15] D. Colton and R. Kress, Looking back on inverse scattering theory, SIAM Rev. 60 (2018), 779-807.

[16] M. Di Cristo and L. Rondi, Examples of exponential instability for inverse inclusion and scattering problems, Inverse Problems 19 (2003), 685-701.

[17] G. Cybenko, Approximation by superpositions of a sigmoidal function, Mathematics of control, signals and systems 2 (1989), 303-314.

[18] H. Dong, D. Zhang and Y. Guo, A reference ball based iterative algorithm for imaging acoustic obstacle from phaseless far-field data, Inverse Probl. Imaging 13 (2019), 177-195.

[19] W.N. E, C. Ma and L. Wu, Barron spaces and the compositional function spaces for neural network models, (2019), https://arxiv.org/abs/1906.08039v1.

[20] F. Girosi and T. Poggio, Networks and the best approximation property, Biological cybernetics 63 (1990), 169-176.

[21] F. Girosi, M.B. Jones, and T. Poggio, Regularization theory and neural networks architectures, Neural computation 7 (1995), 219-269.

[22] X. Glorot and Y. Bengio, Understanding the difficulty of training deep feedforward neural networks, in: Proceedings of the thirteenth international conference on artificial intelligence and statistics, pp. 249-256, Sardinia, Italy, 2010.

[23] I. Goodfellow, Y. Bengio and A. Courville, Deep learning, MIT Press, 2016.

[24] R. Griesmaier, Multi-frequency orthogonality sampling for inverse obstacle scattering problems, Inverse Problems 27 (2011), 085005, 23pp.

[25] G.E. Hinton, S. Osindero and Y. Teh, A fast learning algorithm for deep belief nets, Neural Comput. 18 (2006), 1527-1554.

[26] G.E. Hinton and R. Salakhutdinov, Reducing the dimensionality of data with neural networks, Science 313 (2006), 504-507.

[27] S. Hochreiter and J. Schmidhuber, Long short-term memory, Neural Computation 9 (1997), 1735-1780. 
[28] K. Hornik, M. Stinchcombe and H. White, Multilayer feedforward networks are universal approximators, Neural networks 2 (1989), 359-366.

[29] M. Ikehata, E. Niemi and S. Siltanen, Inverse obstacle scattering with limited-aperture data, Inverse Probl. Imaging 1 (2012), 77-94.

[30] X. Ji, X. D. Liu and B. Zhang, Inverse acoustic scattering with phaseless far field data: uniqueness, phase retrieval, and direct sampling methods, SIAM J. Imaging Sci. 12 (2019), 1163-1189.

[31] X. Ji, X. D. Liu and B. Zhang, Target reconstruction with a reference point scatterer using phaseless far field patterns, SIAM J. Imaging Sci. 12 (2019), 372-391.

[32] D.P. Kingma and J. Ba, Adam: A method for stochastic optimization, (2014), https://arxiv.org/abs/1412.6980.

[33] A. Kirsch and N. Grinberg, The Factorization Method for Inverse Problems, Oxford University Press, 2008.

[34] M. V. Klibanov, A. E. Kolesov and D. L. Nguyen, Convexification method for an inverse scattering problem and its performance for experimental backscatter data for buried targets, SIAM J. Imaging Sci. 12 (2019), 576-603.

[35] A. Krizhevsky and I. Sutskever and G. Hinton, ImageNet classification with deep convolutional neural networks, in: Proceeding of 25th International Conference on Neural Information Processing Systems, pp. 1097-1105, Curran Associates Inc, Nevada, 2012.

[36] Y. LeCun, B. Boser, J. S. Denker, D. Henderson, R. E. Howard, W. Hubbard, and L. D. Jackel, Handwritten digit recognition with a back-propagation network, in: Advances in Neural Information Processing Systems, volume 2, Morgan Kaufman, Denver, 1990.

[37] Y. LeCun, L. Bottou, Y. Bengio and P. Haffner, Gradient-based learning applied to document recognition, Neural networks, 86 (1998), 2278-2324.

[38] Y. LeCun, K. Kavukcuoglu and C. Farabet, Convolutional networks and applications in vision, in: Circuits and Systems (ISCAS), pp. 253-256, Proceedings of IEEE International Symposium, 2010.

[39] M. Leshno, V.Y. Lin, A. Pinkus, and S. Schocken, Multilayer feedforward networks with a nonpolynomial activation function can approximate any function, Neural networks 6 (1993), 861-867.

[40] J. Li and H. Liu, Recovering a polyhedral obstacle by a few backscattering measurements, Journal of Differential Equations 259 (2015), 2101-2120.

[41] J. Li, P. Li, H. Liu and X. Liu, Recovering multiscale buried anomalies in a two-layered medium, Inverse Problems 31 (2015), 105006.

[42] J. Li, H. Liu, Q. Wang, Fast imaging of electromagnetic scatterers by a two-stage multilevel sampling method, Discrete Contin. Dyn. Syst. Ser. S 8 (2015), 547-561.

[43] J. Li, H. Liu and Y. Wang, Recovering an electromagnetic obstacle by a few phaseless backscattering measurements, Inverse Problems 33 (2017), 035011.

[44] J. Li, H. Liu and J. Zou, Strengthened linear sampling method with a reference ball, SIAM J. Sci. Comput. 31 (2009), 4013-4040.

[45] J. Li, H. Liu and J. Zou, Locating multiple multiscale acoustic scatterers, SIAM Multiscale Model. Simul. 12 (2014), 927-952.

[46] P. Li, Y. Wang, Z. Wang and Y. Zhao, Inverse obstacle scattering for elastic waves, Inverse Problems 32 (2016), 115018.

[47] H. Liu, M. Petrini, L. Rondi and J. Xiao, Stable determination of sound-hard polyhedral scatterers by a minimal number of scattering measurements, J. Differential Equations 262 (2017), 1631-1670.

[48] X. Liu, A novel sampling method for multiple multiscale targets from scattering amplitudes at a fixed frequency, Inverse Problems 33 (2017), 085011.

[49] X. Liu and J. Sun, Data recovery: from limited-aperture to full-aperture, (2018), https://arxiv.org/abs/1708.03029v3.

[50] R.D. Mager and N. Bleistein, An approach to the limited aperture problem of physical optics far field inverse scattering, Tech. Report Ms-R-7704, University of Denver, Denver, CO, 1977.

[51] R.D. Mager and N. Bleistein, An examination of the limited aperture problem of physical optics inverse scattering, IEEE Trans, Antennas Propag. 26 (1978) 695-699.

[52] W. Mclean, Strongly Elliptic Systems and Boundary Integral Equation, Cambridge University Press, Cambridge, 2000. 
[53] R. L. Ochs, Jr., The limited aperture problem of inverse acoustic scattering: Dirichlet boundary conditions, SIAM J. Appl. Math. 47 (1987), 1320-1341.

[54] L. Rondi, Stable determination of sound-soft polyhedral scatterers by a single measurement, Indiana Univ. Math. J 57 (2008), 1377-1408.

[55] D.E. Rumelhart, G.E. Hinton and R.J. Williams, Learning representations by back-propagating errors, Nature 323 (1986), 533-536.

[56] U. Shaham, A. Cloninger and R.R. Coifman, Provable approximation properties for deep neural networks, Appl. Comput. Harm. Anal. 44 (2018), 537-557.

[57] A. V. Smirnov, M. V. Klibanov and L. H. Nguyen, On an Inverse Source Problem for the Full Radiative Transfer Equation with Incomplete Data, SIAM J. Sci. Comput. 41 (2019), B929-B952.

[58] H.Y. Sun and L. Demanet, Extrapolated full waveform inversion with deep learning, (2019), https://erlweb.mit.edu/extrapolated-full-waveform-inversion-deep-learning-0.

[59] D. Zhang and Y. Guo, Uniqueness results on phaseless inverse acoustic scattering with a reference ball, Inverse Problems 34 (2018), 085002.

[60] D. Zhang, Y. Guo, J. Li and H. Liu, Retrieval of acoustic sources from multi-frequency phaseless data, Inverse Problems 34 (2018), 094001.

[61] Z. Zhou, Machine learning, Qinghua University Press, 2016.

[62] A. Zinn, On an optimisation method for the full- and limited-aperture problem in inverse acoustic scattering for a sound-soft obstacle, Inverse Problems 5 (1989), 239-253.

School of Mathematics, Jilin University, Changchun, China

E-mail address: gaoyu19@mails.jlu.edu.cn

School of Mathematics, Jilin University, Changchun, China

E-mail address: zhangkaimath@jlu.edu.cn 